\author{
John A. Spencer \\ Rosmarie Forstner \\ Teresa M. Cunha \\ Karen Kinkel \\ on behalf of the ESUR Female \\ Imaging Sub-Committee
}

\section{ESUR guidelines for MR imaging of the sonographically indeterminate adnexal mass: an algorithmic approach}

Received: 25 March 2009

Revised: 11 June 2009

Accepted: 5 July 2009

Published online: 4 September 2009

(C) European Society of Radiology 2009

J. A. Spencer $(\bowtie)$

Department of Radiology,

St. James's University Hospital,

Beckett Street, Leeds, LS9 7TF, UK

e-mail: johnaspencer50@hotmail.com

Tel.: +44-113-2066140

R. Forstner

Universitätsinstitut für

Radiodiagnostik,

Müllner Hauptstr. 48, A-5020,

Salzburg, Austria

T. M. Cunha

Department of Radiology,

Hospital Pulido Valente,

Lisbon, Portugal

\section{K. Kinkel}

Clinique des Grangettes,

Geneva University Hospital and

Institut de Radiologie,

7, Chemin des Grangettes, CH1224,

Chêne-Bougeries/Geneva, Switzerland

\begin{abstract}
A significant proportion of adnexal masses detected by sonography are indeterminate. Either their organ of origin is uncertain or it is unclear whether their nature is benign or malignant. MR imaging of the sonographically indeterminate adnexal mass can resolve most of these uncertainties. Most indeterminate masses result from common benign conditions and women with such masses can avoid unnecessary or
\end{abstract}

inappropriate surgery. For the minority of women whose masses are malignant, use of MR imaging rather than a 'wait and watch' strategy of repeat ultrasound (US) results in a more timely diagnosis. There are simple diagnostic steps in the MR imaging assessment which direct an algorithmic and problem-solving approach based on signal characteristics and morphology. MR imaging should provide a more timely diagnosis and, thereby, guide the management of the patient with reduced costs of investigation and treatment.

Keywords Ovary · Ultrasound · MR imaging $\cdot$ Tumour $\cdot$ Oncology

\section{Introduction}

Ultrasound (US) is the first-line imaging study of women clinically suspected to have an adnexal mass [1]. Combined with clinical assessment and consideration of the serum level of CA-125, US is used to place adnexal masses into one of three categories which define further investigation and management:

- A benign mass

- A malignant mass with or without signs of peritoneal metastasis

- An indeterminate mass

The sonographically (US) indeterminate adnexal mass is defined as one which has complexity but which, after thorough interrogation including Doppler assessment, cannot be confidently placed into either the benign or malignant category; or one for which the site of origin, from the ovary, uterus or another pelvic structure remains to be established.

Adnexal masses are increasingly discovered in the investigation of other abdominal and pelvic problems as crosssectional imaging has supplanted contrast studies of the genitourinary and gastrointestinal tract (e.g. CT colonography and urography, unenhanced CT for detection of urinary calculi) [2-4]. Such incidental findings result in further utilisation of imaging to allay patient and physician anxiety about their nature with significant economic consequences [3].

The clinical impact of defining whether an indeterminate mass is benign or malignant is enormous. Women with malignant adnexal masses require radical cytoreductive surgery by a specialist surgeon with expertise in gynaecological oncology [5-7], whilst benign masses may be either managed conservatively or undergo resection under the care of a general gynaecologist. Further, women with suspected malignancy may require transfer of their care to a specialist institution.

It is no longer appropriate for women with indeterminate masses to undergo exploratory surgery without clear and directed therapeutic intent. For women with these indeterminate masses MR imaging has the capability to:

- Reduce the number undergoing unnecessary surgery for benign lesions - 'overoperation'

- Reduce the risk of missing malignant lesions'underoperation' 
The majority of sonographically (US) indeterminate masses are not rare and exotic lesions but common adnexal lesions $[8,9]$ such as mature teratomas (dermoid tumours) with minimal fat or other atypical features, haemorrhagic lesions in which adherent blood clot may mimic mural vegetations and solid fibrous masses (ovarian fibroma/thecoma and uterine leiomyomata) whose solid nature raises concerns for malignancy. Thus most indeterminate adnexal masses are actually complex benign lesions. Even with application of exemplary MR imaging technique some masses remain indeterminate in nature. It is usual to report such masses as probably malignant. However, the proportion of sonographically indeterminate adnexal masses 'overcalled' as malignant falls dramatically after MR imaging. Less than one in ten indeterminate masses are 'overcalled' by the combination of US followed by MR imaging [8].

There is heterogeneity in this group of indeterminate lesions and a single diagnostic imaging protocol is less easy to define than for other recognised applications of gynaecological MR imaging e.g. for staging malignancy when both the site and histology of disease has already been defined. It is for this reason that the European Society of Urogenital Radiology (ESUR) Female Imaging SubCommittee has discussed and developed an algorithmic approach to the MR imaging assessment of the US indeterminate mass.

\section{Process}

These guidelines were developed by consensus, based on expert opinion and following review of current practice across senior members of ESUR. Following a review of then current literature, two authors (JAS, RF) constructed a questionnaire regarding clinical imaging practice, notably the MR imaging technique used for the indeterminate adnexal mass. An imaging algorithm and draft manuscript were developed and discussed at two meetings of the subcommittee and finalised by the four authors after oral presentation of an outline proposal at the ESUR 2008 meeting (JAS) and a further literature review.

In this article we present our guidelines, illustrating these with images of a series of masses which presented to one of the authors (JAS) in a gynaecological oncology unit as possible malignant masses and whose characterisation using US proved difficult.

\section{Basic MR imaging protocol}

For the examination there is minimal patient preparation. Opinions regarding the need for patient fasting are divided with no supporting evidence. There are, however, data showing the value of intravenous (IV) smooth muscle relaxants for improvement in image quality of pelvic MR imaging [10]. An IV cannula should be left in place in case IV injection of a gadolinium contrast agent is required. Imaging should comprise as a minimum:

- A T2-weighted (T2W) sagittal sequence of the pelvis

- A pair of T1-weighted (T1W) and T2W sequences covering the indeterminate adnexal mass in the same orthogonal (axial or coronal) plane with similar slice thickness

Evaluation of the uterus on the sagittal sequence is important to determine the presence and extent of leiomyomata, adenomyosis which may be associated with endometriosis of the ovary, and to evaluate for endometrial thickening.

The second pair of sequences allows precise comparison of the tissue characteristics of the adnexal mass, detail its anatomic location and suggest its organ of origin. The choice of which orthogonal plane is used is left to the discretion and preference of the supervising radiographer/ radiologist. If the mass is noted to lie lateral to the uterus on the initial sagittal $\mathrm{T} 2 \mathrm{~W}$ sequence its relationship to the uterus may be better shown on coronal or coronal oblique imaging, and if it lies above or behind the uterus on axial or axial oblique orientated imaging.

It is important to identify both ovaries, if there has not been prior resection, as definition of two normal ovaries separate from the mass indicates that the mass is either uterine, tubal or even non-gynaecological. Identification of the ovaries may be aided by a T2W sequence angled along the long axis of the uterine body parallel to the endometrial stripe, along the 'ovarian axis'. This is planned from the $\mathrm{T} 2 \mathrm{~W}$ sagittal sequence of the pelvis. On this sequence the ovaries typically lie lateral to the uterine cornua and below the iliac bifurcation on the pelvic sidewall though there is variation in their location (Fig. 1).

\section{The algorithmic approach: the decision tree}

The decision tree in Fig. 2 divides indeterminate masses into three groups on the basis of their dominant characteristic on the basic $\mathrm{T} 1 \mathrm{~W}$ and $\mathrm{T} 2 \mathrm{~W}$ sequences. For the purposes of this algorithm, solid material has similar signal intensity to muscle on T1-weighted sequences and cyst contents have similar signal intensity on T2-weighted sequences to urine. The three categories of mass are:

- $\quad T 1$ 'bright' masses containing T1 high signal intensity

- T2 solid masses either with predominant signal similar to skeletal muscle, T2 'dark' solid masses, or higher than muscle, T2 'intermediate' or mixed signal solid masses

- Complex cystic or cystic-solid masses

This decision tree directs the selection of additional problem-solving MR imaging sequences and the diagnostic process to define the nature of the great majority of indeterminate adnexal masses and importantly to identify that minority masses which should be considered probably or definitely malignant. 
Fig. 1 Use of the 'ovarian axis': a Transvaginal ultrasonography (TVUS) image showing an indeterminate solid left adnexal mass adjacent to the uterus; b initial sagittal T2weighted image of the pelvis indicating the plane for the 'ovarian axis', the long axis of the uterus, parallel to the endometrial stripe (white line) and $\mathbf{c}$, d the mass shown to be a T2

'dark' solid structure in the left ovary (arrow) separate from the uterus, an ovarian fibroma
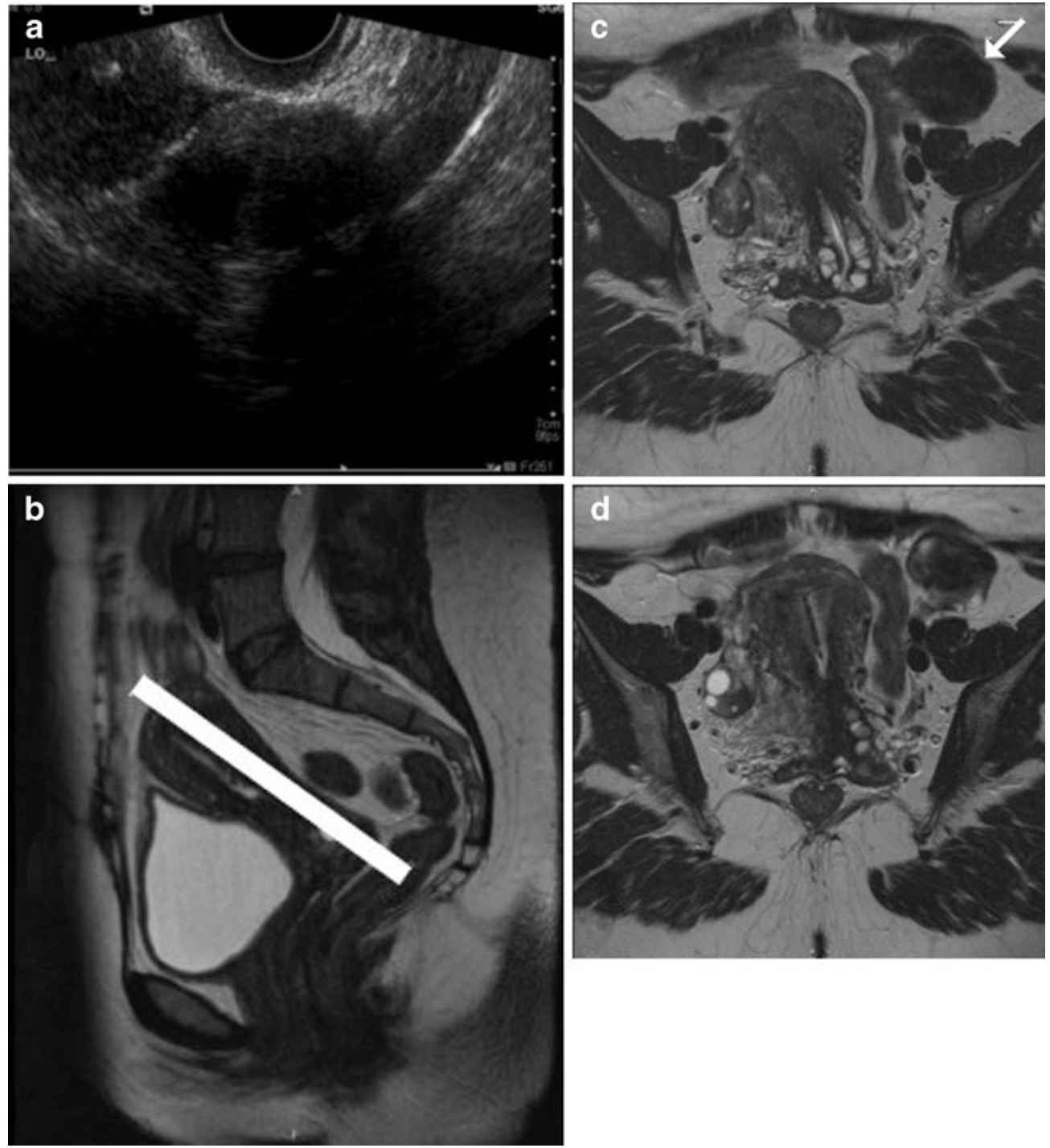

Fig. 2 ESUR decision tree for MR imaging of the US indeterminate adnexal mass. *Note that any mass with MR features suggestive of malignancy should undergo CET1W imaging

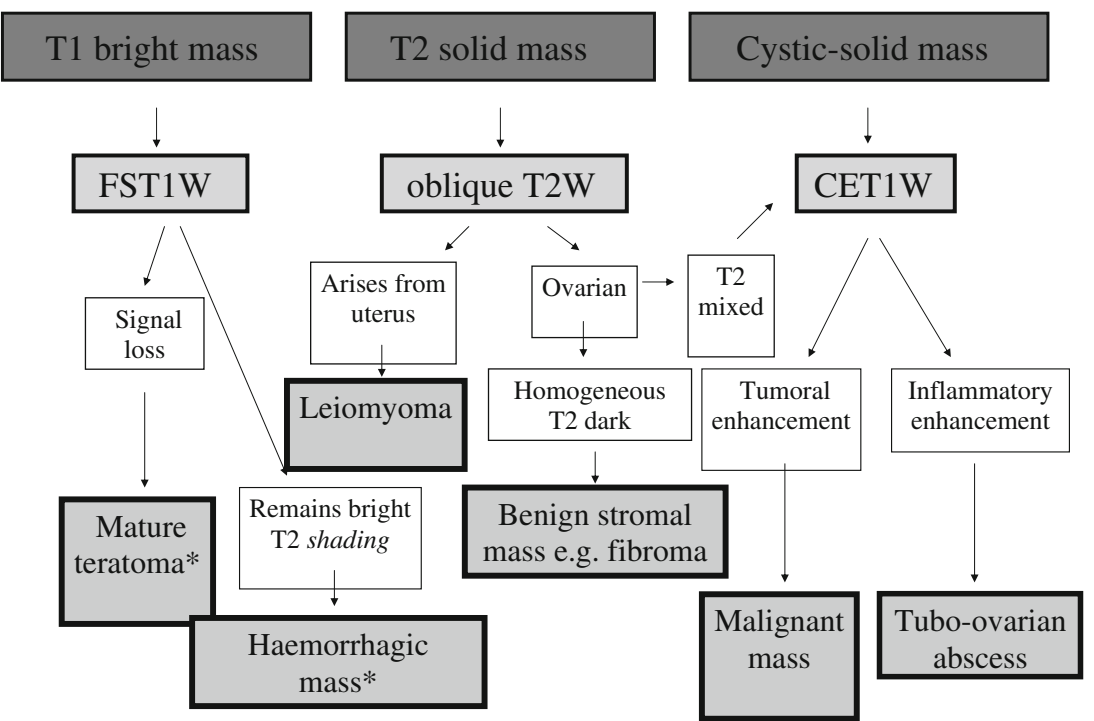


The algorithmic approach: additional MR imaging sequences

Three additional sequences are of value in defining the site of origin and the nature of the indeterminate mass but for most masses a single selected problem-solving sequence suffices to answer the uncertainty of the indeterminate US examination:

- $T 1$ 'bright' masses require additional fat-suppressed T1-weighted (FST1W) imaging using chemical presaturation to distinguish fat in mature teratomas, which show signal loss becoming 'dark' on the FST1W images, from blood, mucin, other proteinaceous material and rarely melanin which remain 'bright'.

- T2 solid masses may require additional T2-weighted oblique imaging across the maximum point of contact of uterus and mass to identify their relationship with the uterus i.e. distinction of uterine leiomyoma from ovarian fibroma. Solid ovarian masses with inhomogeneous low T2 signal or intermediate T2 signal require assessment of the degree of gadolinium enhancement (CET1W).

- All masses with suspected solid elements require gadolinium-enhanced T1-weighted (CET1W) sequences to determine the presence of neoplastic tissue. This applies not only to classic complex cystic and cystic-solid masses but also to other masses with 'worrying' solid components. Some mature teratomas and complex endometriomas contain solid elements which should raise concern as both entities may rarely undergo malignant change.

However, many US indeterminate masses, which are most commonly haemorrhagic or endometriotic cysts, atypical dermoid tumours with minimal fat or in the leiomyoma/fibroma spectrum, can be characterised without recourse to CET1W imaging. Indeterminate adnexal masses are summarised by their dominant signal characteristic in Table 1.

Table 1 Dominant signal characteristic of indeterminate adnexal masses

\begin{tabular}{lll}
\hline T1 'bright' & T2 solid & Cystic-solid \\
\hline Mature teratoma & Leiomyoma & Cystadenoma \\
Haemorrhagic cyst & Fibroma/thecoma & Cystadenofibroma \\
Endometrioma & Struma ovarii & Borderline tumour \\
Mucinous cystadenoma & Primary cancer* & Primary cancer* \\
Melanoma metastasis & Metastasis & Metastasis \\
& & Hydrosalpinx \\
& & Abscess \\
\hline
\end{tabular}

*In the first two decades of life primary cancers are most commonly germ cell or sex cord tumours but in older women are most commonly epithelial tumours

\section{T1 'bright' masses}

These masses require FST1W imaging using chemical presaturation to distinguish fat from blood (Figs. 3 and 4).
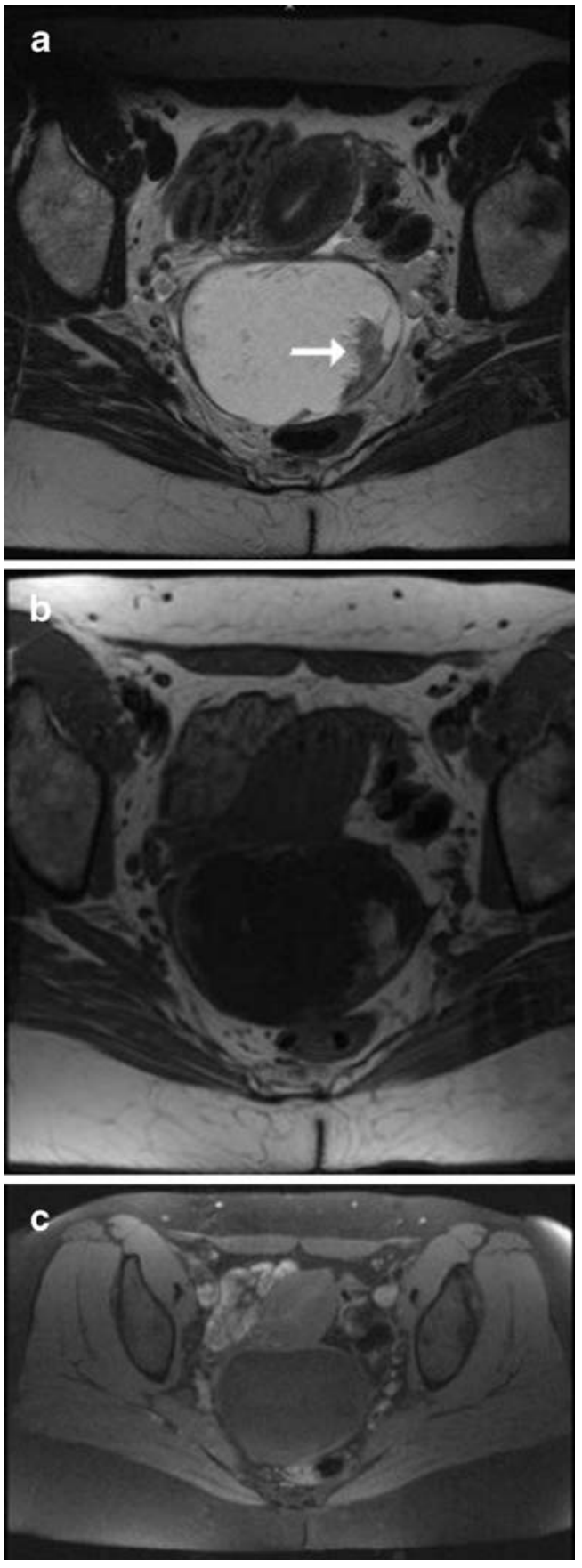

Fig. 3 T1 'bright' mass on MR imaging after US showing a complex cyst with a nodular area in its left wall (arrow): a T2weighted, b T1-weighted and c corresponding fat-suppressed T1weighted image showing complete suppression of the T1 'bright' nodule, a classic mature cystic teratoma with minimal fat. Note on the T2-weighted image subtle chemical shift artefact at the interface of fat and water 
Fig. 4 T1 'bright' mass with MR imaging of haemorrhagic disease of the ovary: a TVUS image showing a complex mass with both hypoechoic and echogenic nodular areas; b T2weighted image showing a left ovary enlarged by T2 'dark' mass which was persistently 'bright' on both the c T1weighted and $\mathbf{d}$ fat-suppressed T1-weighted images. Note the T2 image shows $\mathrm{T} 2 *$ shading in the dependent portion in keeping with sedimentation of chronic blood products in an endometrioma and even on the T1-weighted images some dependent clot causes signal loss (arrow)
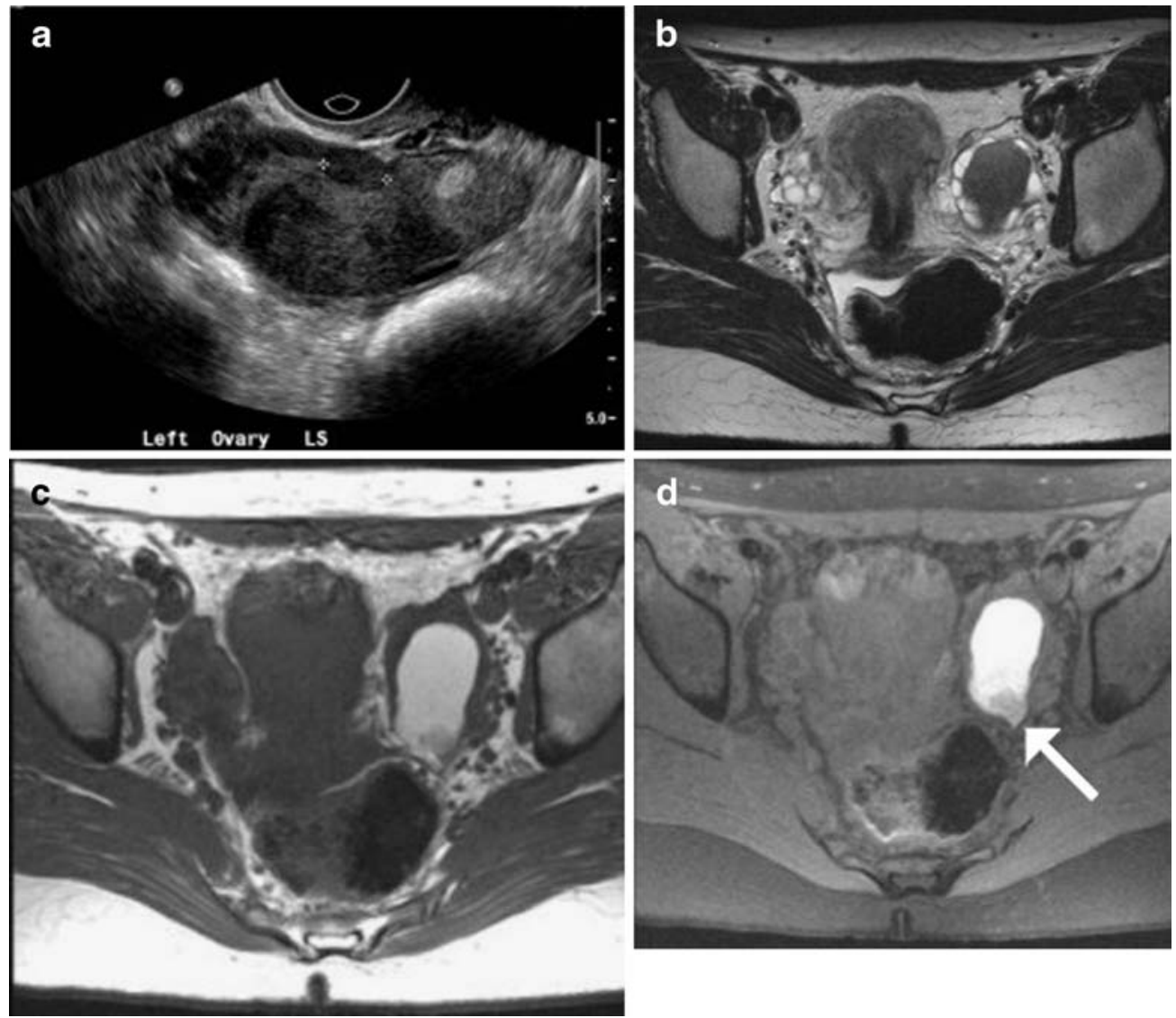

The FST1W sequence should be performed in the same plane as the T1W sequence and with similar parameters to allow direct comparison. Some foci of fat in teratomas are very small but these still show distinct signal loss on the FST1W sequence (Fig. 3).

There are other 'signature' appearances of fat and blood on the standard T1 and T2W sequences. Products of haemorrhage result in $\mathrm{T} 2 *$ effects (Fig. 4) with dependent darkening, dependent graded 'shading' and even brightdark fluid-fluid levels in cysts and/or T2 darkening in the walls of haemorrhagic cysts [11]. Blood products of differing ages and such striking sedimentation and deposition of blood products indicate endometriosis.

Teratomas can have protean appearances. The usual reason why mature teratomas cannot be confidenetly diagnosed by US are a minimal fat content and a predominantly solid nature. The presence of even very small amounts of fat indicates a mature teratoma (Fig. 3) but there may also be blood products from prior torsion of the mass or proteinaceous fluid from glandular components e.g. with secretions from respiratory or enteric-type epithelium (Fig. 5). One should not to be dissuaded from making the diagnosis when there is demonstrable fat suppression by finding these persistently 'bright' areas on the FST1W images. It is important to look for chemical shift artefact (Figs. 3 and 5) which may identify very small fatty elements [12]. It is always important to examine the contralateral ovary carefully for features of a small teratoma as these are commonly bilateral (Fig. 5).

If CET1W images are obtained there may be enhancement of mature elements such as skin appendages which may mimic the features of malignancy. Malignant change in a mature teratoma is quite rare in adults but should be suspected with a dominant solid component showing wall thickening, irregularity or breach. In the childhood/juveniletype teratomas a wide variety of immature and malignant elements may be found. In the older woman with a mature teratoma malignant change is usually from skin elements to squamous carcinoma [13].

With haemorrhagic lesions problems may arise in sonographic distinction of blood clot from a vegetation, notably when this is adherent to the cyst wall. One helpful hint that may be apparent by the time the MR imaging takes place is that the clot has become smaller as further retraction occurs. This is just one reason why comparison with the initial US study is necessary. Again, when there is concern for a solid malignant nodule in a haemorrhagic mass, it must be regarded as a cystic-solid mass which requires IV administration of a gadolinium contrast agent to clarify if the nodule is vascularised tumour. 

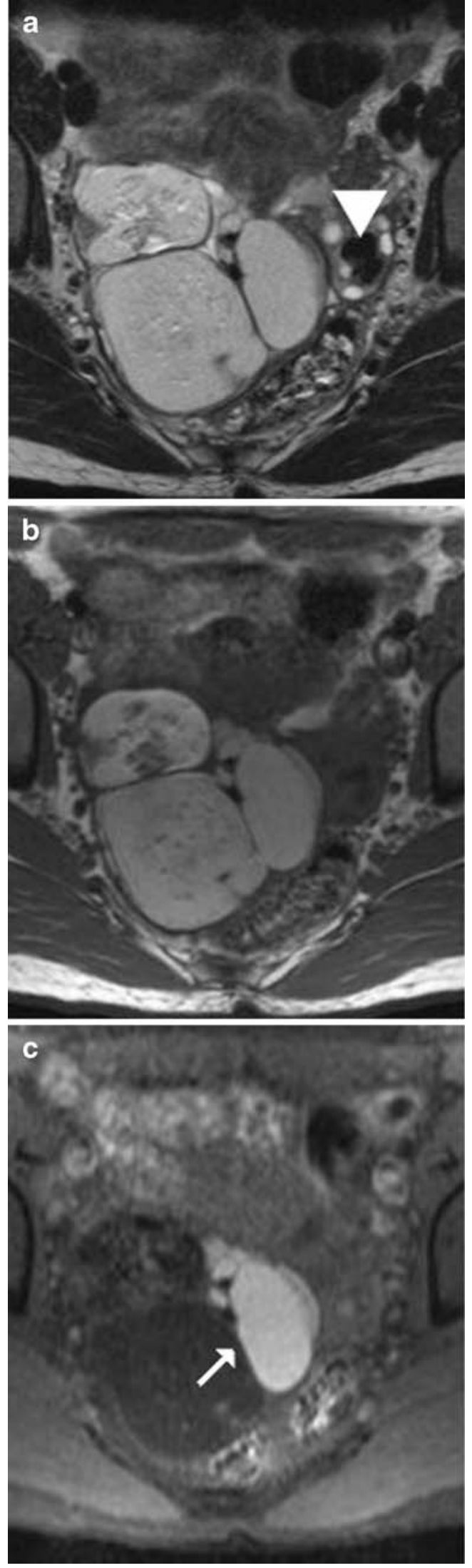

4 Fig. 5 a T2-weighted, b T1-weighted and c corresponding fatsuppressed T1-weighted (FST1W) image showing incomplete suppression of the T1 'bright' lesion, a classic mainly cystic teratoma of the right ovary showing marked internal chemical shift artefact. There is one locule medially which shows no reduction in signal on the FST1W image (arrow) which contained blood products when cut open by the pathologist. Note also small dark nodules in the left ovary (arrowhead) which were calcified/ossified elements in a small fat-poor contralateral mature teratoma

\section{T2 solid masses}

Solid adnexal masses cause alarm because of concerns for metastatic malignancy (Table 1). In clinical practice, however, when dealing with a sonographically solid indeterminate mass without overt signs of malignancy the overwhelming likelihood is of a benign mass. Most are uterine leiomyomata or ovarian fibromata [8]. These are very 'dark' in T2 (and T1) signal when compared with skeletal muscle, often homogeneously black, smooth and well-circumscribed (Fig. 1).

For these masses the algorithmic approach advises oblique $\mathrm{T} 2 \mathrm{~W}$ imaging through the maximum point of contact between the mass and the uterus (Fig. 1). An ovarian fibroma is separate from the uterus and only one normal ovary is seen, contralaterally (Fig. 1). With an ovarian fibroma there may be normal remnant ovary draped around the solid mass or the solid mass may be exophytic from the ovarian tissue [14].

A pedunculated uterine leiomyoma is attached to the uterus by a stalk which may contain the 'bridging vessels' which supply it. Conversely adnexal masses are usually supplied from the gonadal pedicle. These signs of vascular supply may be valuable in further confirming the origin of the indeterminate mass but are not always present. A subserosal uterine leiomyoma has normal myometrium grasping round its margins, the 'claw' sign (Fig. 6). A leiomyoma within the broad ligament is separate from both the uterus and the ovary on that side.

When there is a homogeneous T2 'dark' solid mass which has one of these characteristics diagnosis is completed by use of the oblique $\mathrm{T} 2 \mathrm{~W}$ sequence when this defines the connection or otherwise with the uterus or ovary (Fig. 1).

T2 'intermediate' or mixed signal solid masses have T2 intermediate signal or T2 inhomogeneous signal with a mixture of $\mathrm{T} 2$ dark and bright signal. These may represent either benign solid masses which have undergone degeneration, endocrine active tumours such as thecoma, Leydig cell or granulosa cell tumour or malignant masses, either primary or secondary. The last of these is of greater concern in a woman with a history of a cancer recognised to metastasise to the ovary e.g. bowel or breast cancer.

Whilst classic T2 'dark' solid masses shown to be ovarian fibromata do not require further characterisation, T2 'intermediate' or mixed signal solid masses may require 


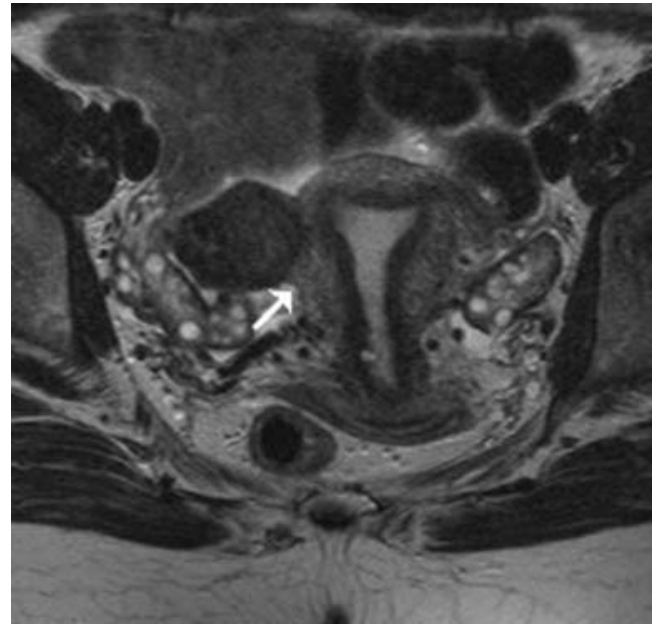

Fig. 6 T2 'dark' solid mass with oblique T2W MR imaging along the 'ovarian axis' showing a subserosal leiomyoma of the right side of the uterus (arrow) with a 'claw' of normal myometrium grasping it. Note the normal right ovary lateral to the mass and from which it is quite separate

CET1W imaging (Figs. 7 and 8). Concern about a solid mass may be furthered when there is ascites but this does not necessarily imply malignancy as it may be seen with some fibromata (Meig's syndrome).
After IV injection of gadolinium benign lesions in the fibrothecoma spectrum show little or no enhancement (Fig. 7) but solid malignant masses show marked enhancement (Fig. 8). Thus masses with similar T2 signal and appearances are easily separated into benign or malignant categories by additional CET1W imaging (Figs. 7 and 8). The difference in enhancement is usually dramatic. A minority of our group recommended use of fat-suppressed CET1W imaging to assess the degree of enhancement (Fig. 7) but there are no convincing data to support this. Indeed data are lacking to define just how much enhancement should be considered significant.

Endocrine tumours with functioning stroma are rare. These more commonly present clinically with hormonal effects such as hirsutism (Leydig cell tumour) or uterine oestrogenisation with endometrial thickening or dysfunction (granulosa cell tumour). Hence these are more often discovered in a search for the cause of the hormonal problem than as a sonographically indeterminate mass. These tumours have a variety of morphological features but usually show significant enhancement [15].

Some malignant masses are predominantly solid (Fig. 8). On CET1W imaging these enhance brightly in their solid components but may show necrosis as the tumour outgrows its blood supply. Women with solid malignant masses require referral to a specialist gynaeco-
Fig. 7 Use of CET1W imaging for a T2 'intermediate' solid mass: a TVUS image showing a solid adnexal mass and $\mathbf{b}$ sagittal T2-weighted of the pelvis showing an intermediate signal inhomogeneous mass (arrow) separate from the uterine fundus and small volume ascites in this post-menopausal woman; c T1weighted and $\mathbf{d}$ fat-suppressed contrast-enhanced T1-weighted image showing minimal enhancement (arrowhead) when compared with the uterus (arrow). This was a fibroma with a minimal thecoma element
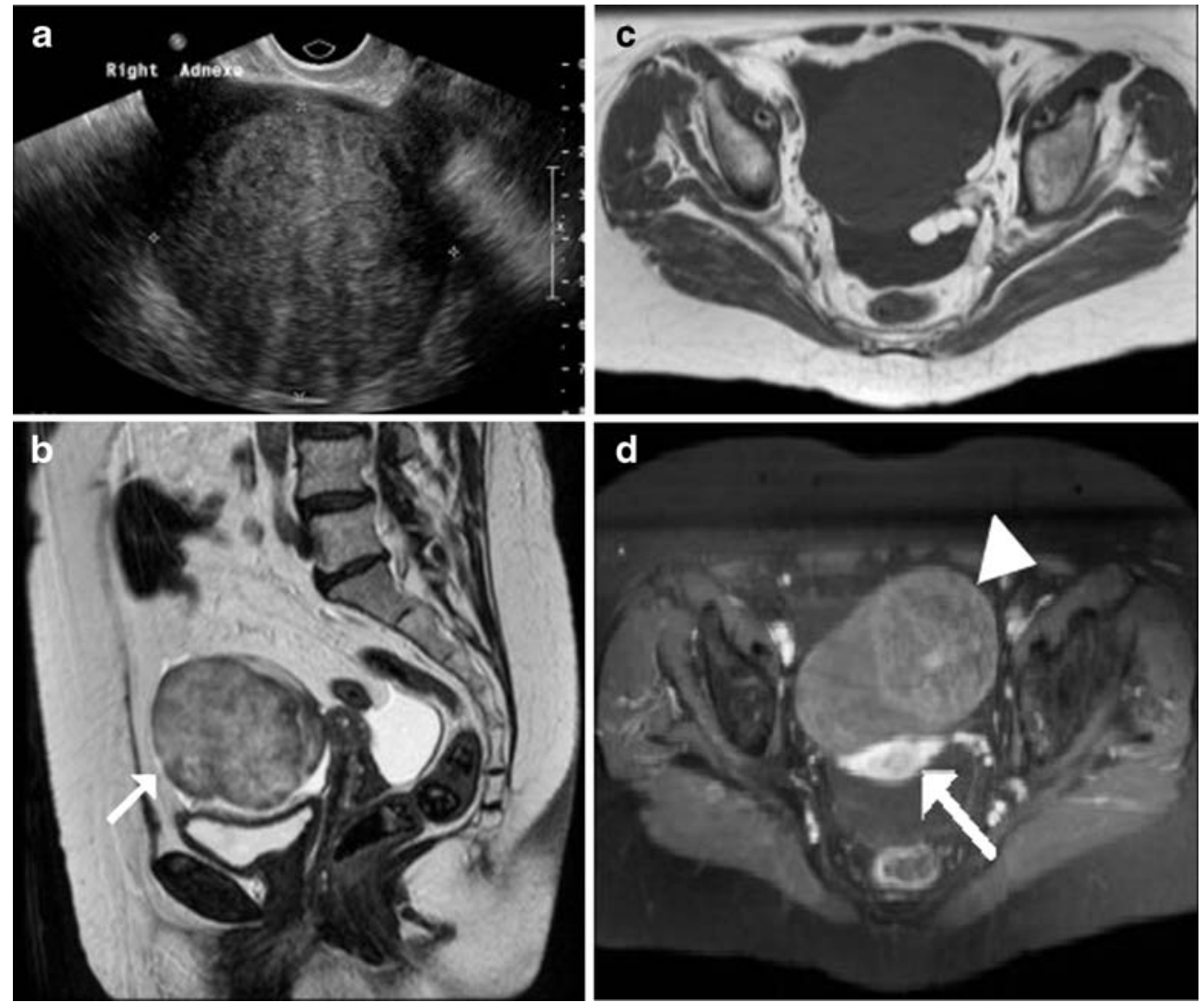

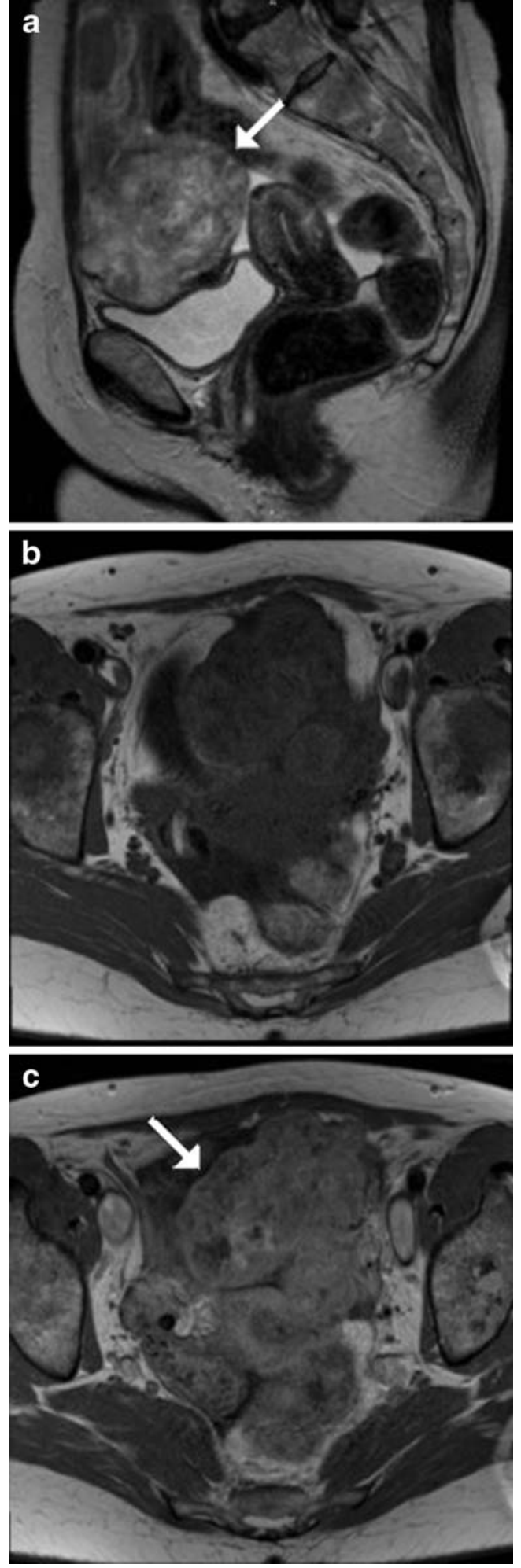

logical oncologist and should undergo further assessment using contrast-enhanced CT (CECT). With suspected ovarian cancer CECT of the abdomen and pelvis is recommended to assess the extent of disease but also to
4 Fig. 8 Use of CET1W imaging for a T2 'intermediate' solid mass: a sagittal T2-weighted of the pelvis showing an intermediate signal inhomogeneous mass (arrow) separate from the uterine fundus and small volume ascites in this post-menopausal woman; b T1weighted and c contrast-enhanced T1-weighted image showing marked enhancement (arrow) with some areas of necrosis within the solid mass. This was a solitary metastasis from a previously resected breast cancer

inspect sites of potential primary tumour in the gastrointestinal tract. Some primary epithelial ovarian cancers are solid but the concern for any solid malignant mass should also be for a metastasis from sites such as the bowel or breast (Fig. 8).

Post-menopausal ovaries are now commonly identified by modern high resolution cross-sectional imaging. It is no longer true that, if identified by cross-sectional imaging, post-menopausal ovaries are probably enlarged and thus abnormal. They are now often seen with high resolution US and CT. At MR imaging post-menopausal ovaries are predominantly solid with a stroma which is T2 'dark' in signal and which may contain small immature follicles. The solid stroma does not enhance.

\section{The complex cystic or cystic-solid mass}

The most common benign masses which fall within this category are multilocular benign cysts, complex tubal disease and cystadenofibromata. The most worrying diagnosis is ovarian malignancy, either borderline or frankly invasive cancer.

Purely cystic lesions are benign and do not require CET1W images, likewise chronic hydrosalpinx, whose convoluted and opposed folds may cause US findings which mimic thickened septa. Conversely, acutely tuboovarian masses may produce very complex masses with wall thickening and 'pseudo-solid' areas. These enhance brilliantly and can mimic malignancy. The clinical features of acute pelvic infection and malignancy usually differ, though some chronic infections such as tuberculosis and actinomycosis may exactly mimic malignancy [16].

The usual cause of an US indeterminate cystic mass is an area of wall thickening or irregularity. For most cases simple pre- and post-gadolinium $\mathrm{T} 1 \mathrm{~W}$ sequences suffice to evaluate enhancement in nodular mural components (vegetations) (Fig. 9). Larger solid areas in malignant masses may show necrosis (Fig. 8) [17, 18]. Enhancement may be seen in benign cystic-solid lesions in the adenofibroma-cystadenofibroma spectrum (and may correlate with endocrine activity) but these usually show distinct low T2 signal [18].

Another option for cystic-solid masses is the use of dynamic contrast-enhanced MR imaging. This technique can be 'temporally bracketed' between the T1W and CET1W sequences. Recent data suggest that enhancement profiles within solid vegetations can help in identifying 
Fig. 9 CET1W MR imaging of a small stage IA ovarian cancer: a referral colour Doppler TVUS image of a complex cystic-solid mass with internal blood flow, an unexpected finding in a 29 year-old woman considered to have endometriosis; b sagittal T2-weighted and axial pre- (c) and post-contrast (d) T1weighted images showing intense enhancement of the internal nodules (arrows). After US a malignant diagnosis was considered as well as complex endometriosis but after MR imaging discussion fertility sparing cancer surgery was planned in this young woman
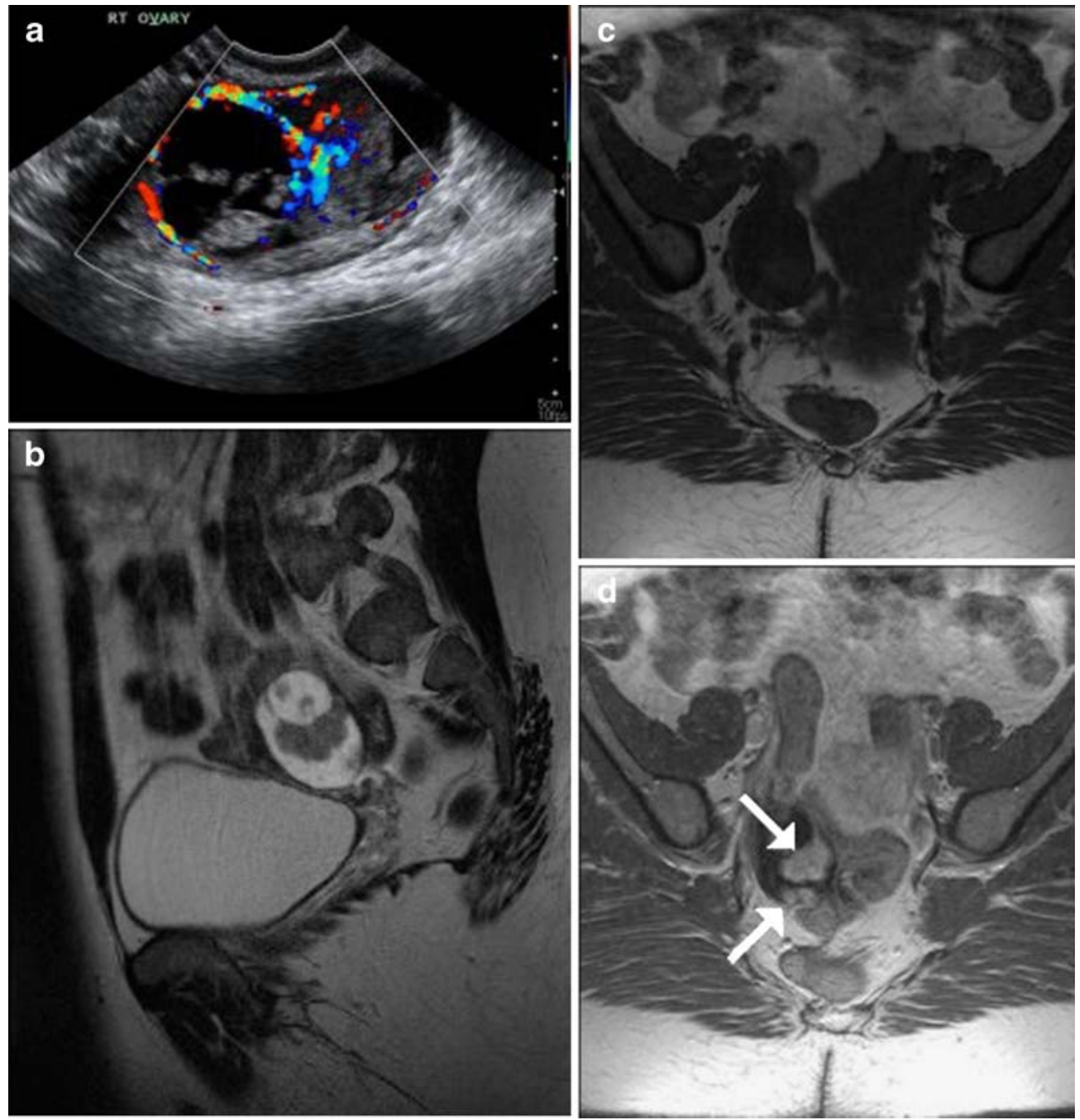

lesions which require cancer surgery by distinction of benign from borderline and malignant disease [19].

In some cases the findings of US are highly likely to represent malignancy but the finding is totally unexpected e.g. in a very young woman (Fig. 9). Here MR imaging can be used for secondary confirmation. Even very small foci of malignancy can be demonstrated by CET1W MR imaging (Fig. 10).

\section{Practical considerations}

The principal strength of this algorithmic approach (Fig. 2) also points to a practical difficulty. Supervision of the examination by a radiologist with an interest in gynaecological imaging may be required to define into which category to place the mass, which problem-solving sequences need to be performed and for some cases in which plane to perform problem-solving sequences. We recognise that this supervision of pelvic MR imaging though desirable is not universally available.

We would argue that this application of MR imaging has such importance in determining clinical management that it deserves such supervision and should be separated from routine protocol-driven applications of MR imaging. If this cannot be achieved all the problem-solving sequences may need to be performed. Nonetheless the diagnostic discipline of the algorithm should assist in arriving at a clinically useful answer to the problem. Experienced MR radiographers/technologists using the algorithm soon become skilled at recognising the MR imaging patterns and characteristics of the common indeterminate masses and in selecting appropriate sequence(s) to solve the problem. The MR imaging protocol is summarised in Table 2 and the decision tree in Fig. 2.

In principle, an earlier diagnosis of cancer should result from the use of MR imaging. A commonly used sono- 
Fig. 10 CET1W MR imaging of a tiny stage IA borderline ovarian cancer: a TVUS image showing a small solid nodule within the right ovary and $\mathbf{b} \mathrm{T} 2-$ weighted, c T1-weighted and d CET1-weighted images showing enhancement of the small nodule (arrow). This 54-yearold woman had lower abdominal discomfort and a slightly raised CA-125. The TVUS diagnosis was of follicular haemorrhage
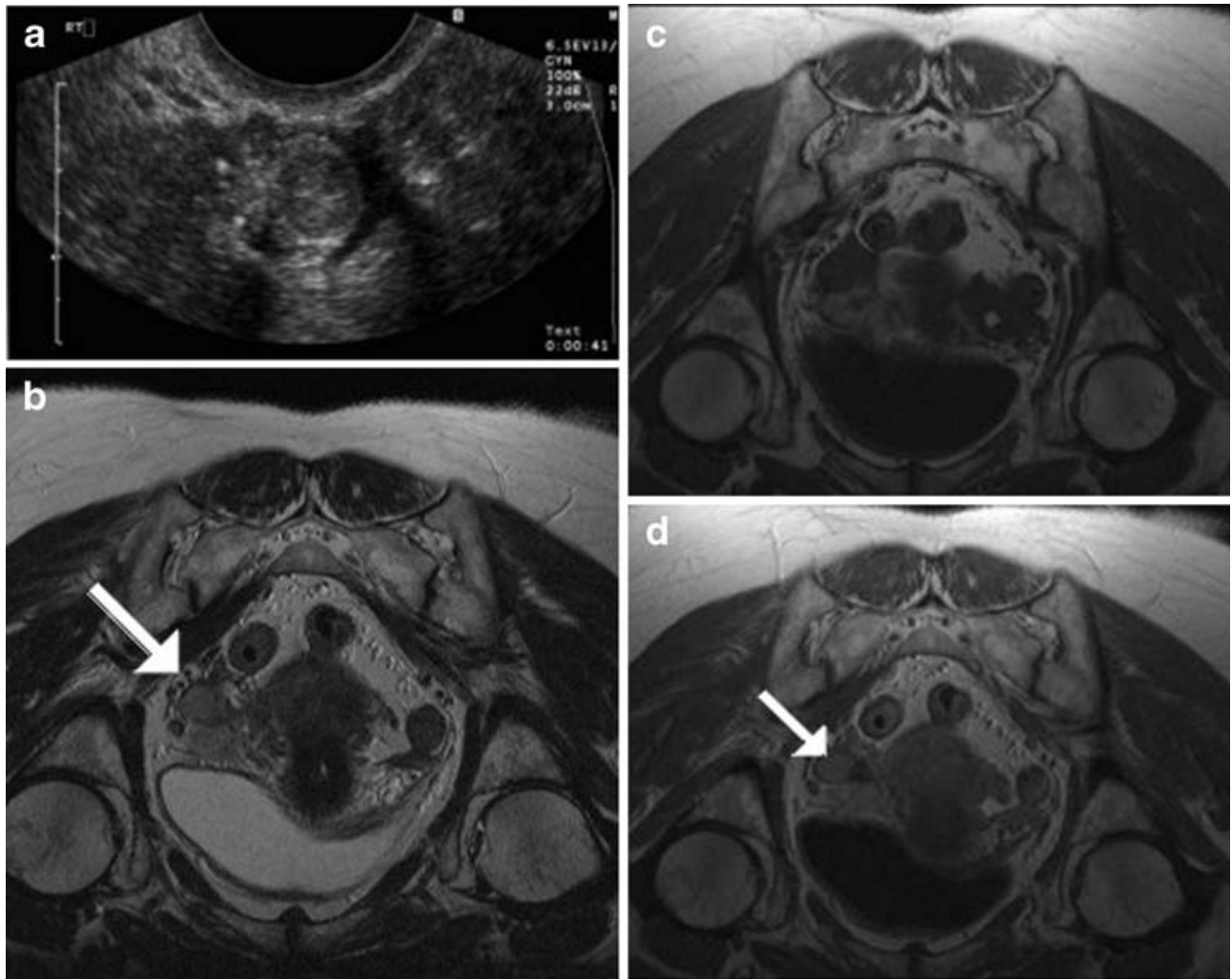

graphic strategy for the indeterminate mass has been to repeat the examination after two or more menstrual cycles to allow products of haemorrhage, which can mimic solid tumour, to resolve. With MR imaging this delay of 2 to

Table 2 Summary of MR imaging protocol

Patient preparation Intravenous smooth muscle relaxant Placement of intravenous cannula

Basic MR Sagittal T2W of the pelvis

sequences Pair of T1W, T2W through the indeterminate mass

$\pm \mathrm{T} 2 \mathrm{~W}$ sequence in the long axis of the uterus*

Problem-solving T1 'bright' mass-FST1W

sequences $\quad \mathrm{T} 2$ 'dark' solid mass-oblique T2W*

T2 solid mass-CET1W

Cystic-solid mass-CET1W

*In many cases this oblique $\mathrm{T} 2 \mathrm{~W}$ sequence along the 'ovarian axis' suffices (Fig. 1). In other cases a plane selected across the maximum point of contact of the mass and uterus is required to see if it is ovarian or uterine in origin
3 months can be avoided and the timely diagnosis fits well within a rapid cancer diagnosis strategy [20].

\section{Summary}

Most US indeterminate masses which undergo MR imaging are complex benign lesions. The intervention of MR imaging can reduce the number of women undergoing unnecessary 'overoperation' as it allows identification of benign features that obviate cancer surgery. Conversely an indeterminate adnexal mass with abnormal solid enhancing (CET1W) soft tissue elements should be viewed as a cancer, referred to a specialist surgeon and undergo staging and further assessment within a specialist centre. MR imaging allows discrimination of tiny malignant elements within ovarian masses (Fig. 10).

MR imaging has maximal impact when CA-125 levels are normal or only slightly raised (Fig. 10) [8]. It has a central role in defining management options for women suspected to have ovarian cancer [21]. It is the most costeffective intervention for the sonographically indeterminate adnexal mass with the greatest shift in pre-test probability [22]. 
A small minority of masses will be incorrectly diagnosed using this algorithmic approach to MR imaging. In series reported so far these were 'false positive' diagnoses of cancer but this occurred in less than one case in ten [8]. Fatpoor teratomas, atypical fibromas and cystadenofibromas [18] continue to confound pre-operative MR imaging assessment.
Acknowledgements We are grateful to the following members of the subcommittee who contributed to discussion of the guidelines and responded to a questionnaire about current practice in their institutions: B Hamm, C Lopez, K Togashi, C Balleguier, R KubikHuch, R Manfredi, C Nicolao, AC Tsili and M Bazot. JAS is also grateful to Mrs Sheila Boyes, Ms Fiona Lang and Dr Sunethra Ghattameneni for help with selection and preparation of images to illustrate this article and for helpful comments on the manuscript.

\section{References}

1. Royal College of Radiologists (RCR) (2007) Making the best use of clinical radiology services, 6th edn. RCR, London

2. Xiong T, Richardson M, Woodroffe R, Halligan S, Morton D, Lilford RJ (2005) Incidental lesions found on CT colonography: their nature and frequency. Br J Radiol 78:22-29

3. Pickhardt PJ, Hanson ME, Vanness DJ et al (2008) Unsuspected extracolonic findings at screening CT colonography: clinical and economic impact. Radiology 249:151-159

4. Katz DS, Scheer M, Lumerman JH, Mellinger BC, Stillman CA, Lane MJ (2000) Alternative or additional diagnoses on unenhanced helical computed tomography for suspected renal colic: experience with 1000 consecutive examinations. Urology 56:53-57

5. Earle CC, Schrag D, Neville BA et al (2006) Effect of surgeon specialty on processes of care and outcomes for ovarian cancer patients. J Natl Cancer Inst 98:172-180

6. Engelen MJ, Kos HE, Willemse PH et al (2006) Surgery by consultant gynecologic oncologists improves survival in patients with ovarian carcinoma. Cancer 106:589-598
7. Vernooij F, Heintz P, Witteveen E, van der Graaf Y (2007) The outcomes of ovarian cancer treatment are better when provided by gynecologic oncologists and in specialized hospitals: a systematic review. Gynecol Oncol 105:801-812

8. Sohaib SA, Mills TD, Sahdev A et al (2005) The role of magnetic resonance imaging and ultrasound in patients with adnexal masses. Clin Radiol 60:340348

9. Adusumilli S, Hussain HK, Caoili EM et al (2006) MRI of sonographically indeterminate adnexal masses. Am J Roentgenol 187:732-740

10. Johnson W, Taylor MB, Carrington $\mathrm{BM}$, Bonington SC, Swindell R (2007) The value of hyoscine butylbromide in pelvic MRI. Clin Radiol 62:1087-1093

11. Nishimura K, Togashi K, Itoh K et al (1987) Endometrial cysts of the ovary: MR imaging. Radiology 162:315-318

12. Outwater EK, Dunton CJ (1995) Imaging of the ovary and adnexa: clinical issues and applications of MR imaging. Radiology 194:1-18

13. Kido A, Togashi K, Konishi I et al (1999) Dermoid cysts of the ovary with malignant transformation: MR appearance. Am J Roentgenol 172:445-449

14. Oh SN, Rha SE, Byun JY et al (2008) MRI features of ovarian fibromas: emphasis on their relationship to the ovary. Clin Radiol 63:529-535
15. Jung SE, Rha SE, Lee JM et al (2005) $\mathrm{CT}$ and MRI findings of sex cordstromal tumor of the ovary. Am J Roentgenol 185:207-215

16. Kim SH, Kim SH, Yang DM, Kim KA (2004) Unusual causes of tubo-ovarian abscess: CT and MR imaging findings. Radiographics 24:1575-1589

17. Hricak H, Chen M, Coakley FV et al (2000) Complex adnexal masses: detection and characterization with MR imaging-multivariate analysis. Radiology 214:39-46

18. Guerra A, Cunha TM, Félix A (2008) Magnetic resonance evaluation of adnexal masses. Acta Radiol 49:700-709

19. Thomassin-Naggara I, Bazot M, Daraï E, Callard P, Thomassin J, Cuenod CA (2008) Epithelial ovarian tumors: value of dynamic contrast-enhanced MR imaging and correlation with tumor angiogenesis. Radiology 248:148-159

20. Department of Health (2000) Referral guidelines for suspected cancer. Stationery Office, London

21. Spencer JA, Forstner R, Hricak H (2008) Investigating women with suspected ovarian cancer. Gynecol Oncol 108:262-264

22. Kinkel K, Lu Y, Mehdizade A, Pelte MF, Hricak H (2005) Indeterminate ovarian mass at US: incremental value of second imaging test for characterization-meta-analysis and Bayesian analysis. Radiology 236:85-94 